\title{
Design of Aerial Panoramic Photography: Contrast between Industrialized and Natural Zones
}

Wei Zhong Feng ${ }^{1}$, Yu-Che Huang ${ }^{2, *}$, Fang-Lin Chao ${ }^{2}$

${ }^{1}$ Department of Visual Communication, Chaoyang University of Technology, Taichung, 436, Taiwan, R.O.C.

${ }^{2}$ Department of Industrial Design, Chaoyang University of Technology, Taichung, 436, Taiwan, R.O.C.

\begin{tabular}{l} 
A R T I C L E I N F O \\
\hline Article history: \\
Received: 06 July, 2020 \\
Accepted: 25 July, 2020 \\
Online: 31 August, 2020 \\
\hline Keywords: \\
Panoramic \\
Aerial film \\
Creation \\
Environmental awareness \\
Planet image
\end{tabular}

\begin{abstract}
A B S T R A C T
This study utilized aerial panoramic photography and $3 D$ virtual reality to demonstrate the environmental changing issues. Environmental problems have occurred in the surroundings for a long time, but the public has gradually become numb. The wide view through aerial photography helps to show the difference. The proposed self-assembled drone equipped with an antishock bracket containing a six-axis aerial camera was used to capture videos with the critical environmental problems in Taiwan. The captured panoramic video was processed using post-production software to create a unique documentary revealing the adverse effects of pollution. The video portrayed a sharp contrast between the eastern and western coastline of Taiwan by presenting planet images. Most participants of a survey reported increased awareness of environmental protection after watching the aerial panoramic films.
\end{abstract}

\section{Introduction}

Taiwan's limited land resources are often annexed by industries to satisfy economic needs. The industrialization has considerably enriched people's lifestyles but also permanently changed the environment. Because changes occur gradually over an area, people habitually endure and ignore them.

Farmlands are gradually being converted into industrial and residential zones. This unbalanced utilization of environmental resources poses a threat to the sustainability of society. Although laws related to environmental protection have been enacted, the conversion of farmland into industrial zones has not abated. Although institutions have attempted to preserve cultivated land [1], recent manifestation of environmental problems indicates that development policies require reform. The education of citizens regarding environmental protection is crucial for the development of sustainability-related social consciousness. Agriculture and health sustainability need public policy support, especially in the context of investment and regional technology development [2].

\subsection{Technology Background}

Aerial panoramic photography and 3D virtual reality technologies are used in artistic creation and movies to demonstrate the environmental changing issues. These technologies contrasted landscape transformed by industrialization

*Corresponding Author: Y.C. Huang, 79 Futong St., Taichung 436, Taiwan R.O.C., 886-910673898, hyche@ cyut.edu.tw to evoke reflection on the sustainable utilization of resources. Panoramic art has been employed to present ecological changes to the public to induce such reflection. Dramatic representation using artistic techniques causes people to think about the relationship between humans and the environment in the process of modernization. People's perception helps to identify and weigh social priorities and maintain desired sustainable relationships.

New technology allows us to experience situations from a firsthand perspective. To achieve this goal, the following techniques were used to present design concepts:

- Aerial photography: Aerial photography provides a broad view of the changes in a landscape. However, aerial photography cannot offer a microscopic perspective of the changes.

- Digital image processing: This processing technology enables us to show the overall relationship. Post-production can considerably transform captured scenes; for example, a remote $360^{\circ}$ perspective can be employed to convey an idea effectively.

- Multiple projectors connected in series: Images can be divided to depict various perspectives to prompt the viewer's emotional investment.

- Virtual reality (VR) enabled by mobile phones: Mobile phones have built-in gyroscopes and orientation sensors. The 
orientation of mobile phone changes the viewing angle, and the image displayed on the mobile phone screen changes. VR can be used to take advantage of these devices and present extraordinary experiences without the need for additional helmets and induction devices.

VR video technology has emerged as a new medium of documentary filmmaking. More news editing teams are using immersive technologies to relate news events effectively. These teams are usually composed of technical and journalism teams [3]. Although journalism is a time-critical field, limited research has been conducted on immersive technology. VR provides a humancomputer interactive experience and has, therefore, become a new avenue for documentary filmmaking. The appropriate application requires an understanding of advanced interdisciplinary methods and proper design and techniques [4].

The configured aerial imaging platform provides a new perspective and enables the user to investigate high-altitude locations [5]. When a user moves along a high-altitude path, the device receives location data from the camera sensor. Unidentified aerial system (UAS) provides instant processing under controlled conditions. The cameras installed on the platform have the following specifications[6]: GoPro Hero and DJI [7]. UAS images can clearly show areas of concrete and steel bridges that are generally inaccessible. Furthermore, non-destructive damage can be detected, which is suitable for conveying environmental problems. Observing the structure and dynamics of social ecosystems in specific geographic areas [8] can help policymakers. However, drones pose security risks and privacy concerns [9]. Therefore, the government strictly regulates their use.

\subsection{Related Studies}

Considerable progress has been achieved in the creation of static spherical panoramic images and full-area guided VR tours. These technologies are now user friendly and widely employed [10]. Digital camera technology has evolved considerably over the years, resulting in improved image resolution. Image pixels are no longer the main distinguishing factor of mobile visual communication. User-friendly interconnectivity is essential for creating immersive user experiences and visual interfaces [11]. For example, Google Street View, which provides location photos accumulated through user participation, is a popular platform. Street View is also used in virtual tours, even allowing advanced navigation functions in designated regions.

\section{Concept and implementation}

\subsection{Concept}

Because Taiwan relied on thermal power generation, coupled with industrial intermediate byproducts and vehicle exhaust, fine particles are currently the country's first environmental problem. The high number of suspended particles in the vicinity of thermal power plants is a concern for public health. In Taiwan, thermal power plants are located along the central coast of Taiwan (Figure 1a). However, the considerable suspended matter has been observed in Puli, a mountainous area. A cumulative effect has thus occurred, caused by the accumulation of fine floating particles in the basin area with a range of more than $100 \mathrm{~km}$.

Taiwan's main petrochemical and medium-sized industries are located in Kaohsiung City in southern Taiwan (Figure 1b). The industrial zone and Petro-China Kaohsiung Refinery constitute a major petrochemical town of south Taiwan. This industrial area borders a river in the east, and the Taiwan Strait is located to the south approximately $25 \mathrm{~km}$ from city. The total number of manufacturers in the area is 33, including 19 chemical material and four chemical product manufacturers. The coast was originally an ecological zone full of mangroves but is being slowly converted into an industrial area.

The staggered existence of agricultural and industrial areas is a controversial issue. Agricultural land becomes an option for industrialization when land acquisition is difficult. The sewage discharge and waste treatment of factories in agricultural areas affect the lives of nearby families.

Because of the three aforementioned observations, we selected the following as the focus of our documentary: (1) thermal power plants (Figure 1a), and (2) major petrochemical industrial areas (Figure 1b). Factories scattered in rice fields in suburbs of the city (Figure 1c). Pollution images were captured through aerial photography and used to compare pollution levels in the industrial and agricultural sectors, which could then be presented to the public to understand the situation.

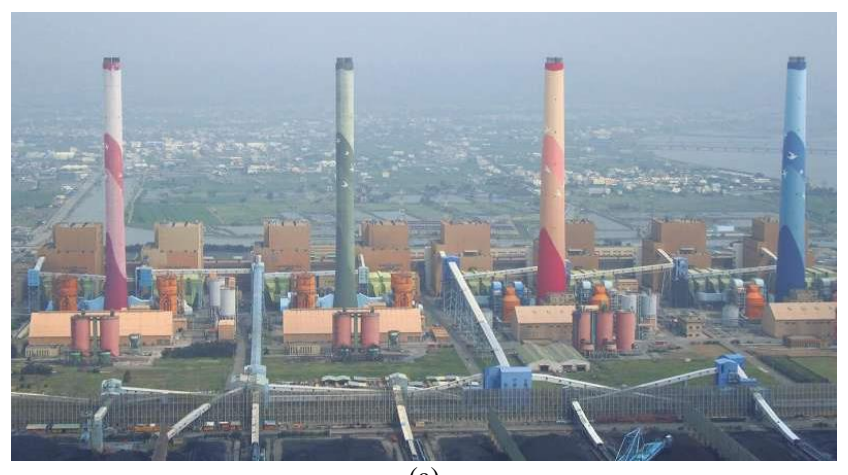

(a)

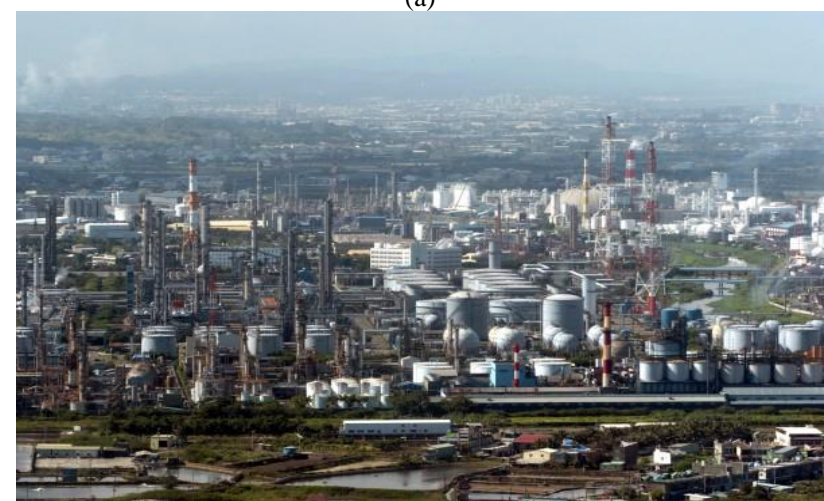

(b)

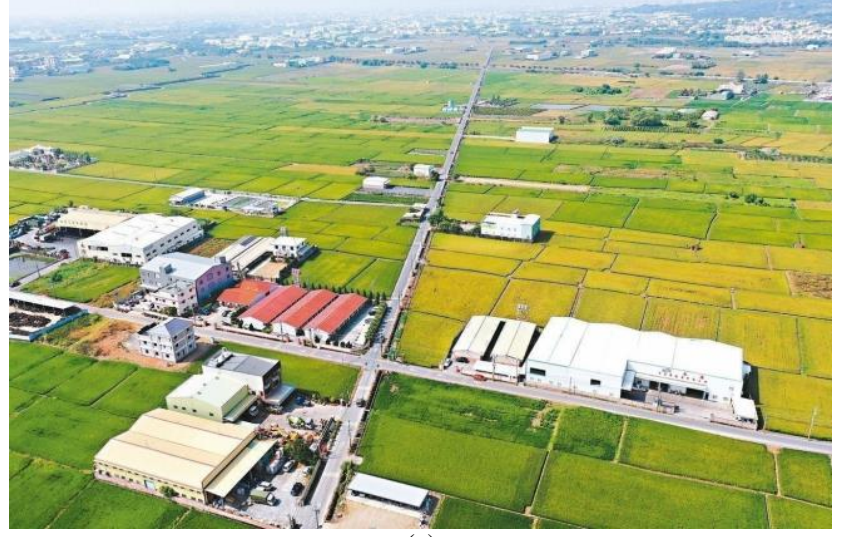

(c)

Figure 1: (a) Thermal power plant, (b) Refinery Industrial Zone, (c) Factories scattered in rice fields 


\subsection{Visual Design Setting}

Figure 2 depicts the design process of image production. An arranged screen spliced by projectors. A shockproof photography bracket was mounted on a drone. GoPro HERO3+ motion recorders (six underneath) were installed. The obtained $4 \mathrm{k}$ panoramic video was then edited using Kolor-Autopano software, with the landscape images transformed into a planet-shaped image displaying a sharp contrast between industrial and agricultural areas.

The video-capturing method of a $360^{\circ}$ panoramic camera is different from that of an ordinary camera. Irrespective of lens rotation and tilting, the picture remains horizontal and faces the same direction. Panoramic shooting mainly provides high and low or left and right viewing angles. The $720^{\circ} \mathrm{VR}$ display is a $360^{\circ}$ horizontal and vertical guide, allowing the user to decide the perspective of the scene or view of the image.

Kolor-Autopano software is a video-editing software program that can be used to stitch together two or more panoramic videos of overlapping areas to correct the horizon control points. A parallax compensation algorithm for stitching videos enables the user to reduce the time required to fix stitching issues in postproduction. A planet-shaped image, called a small planet with a parallel display, is the desired viewing style in power plant projects. These tiny planet photos are a novel means of displaying panoramas and landscape photos.

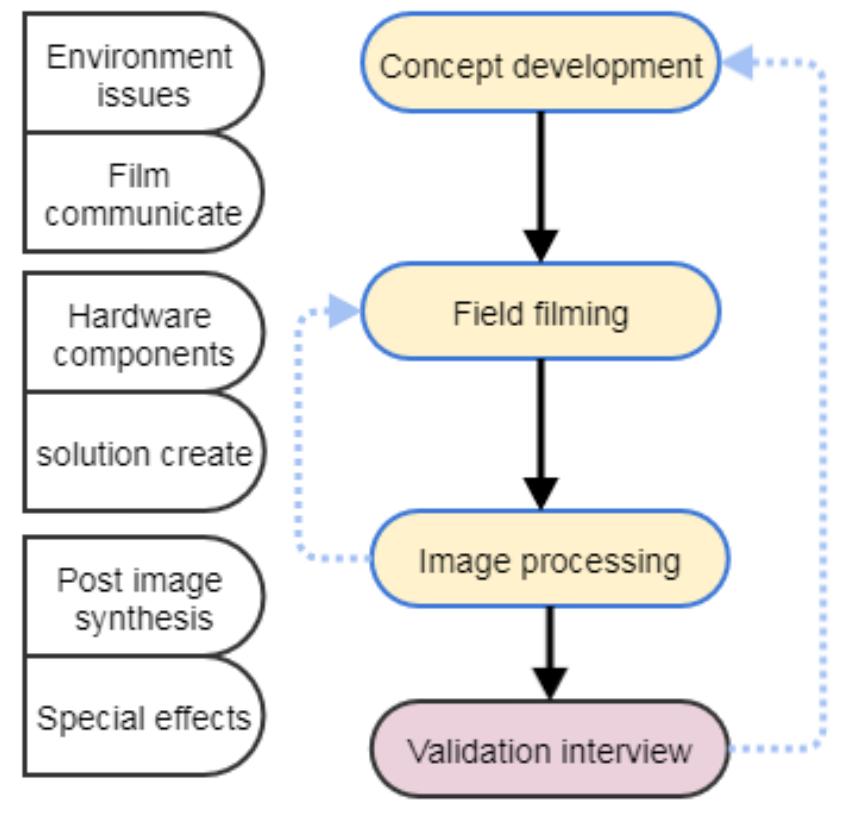

Figure 2: Iterative design and study process.

Hardware implementation (Figure 3) utilized several facilities with the flying drone to ensure the image quality. From the top to the bottom of the fuselage, there are Kodak Pixpro SP360 Camera, three-axis gimbal stabilizer for VR Camera, three-axis gimbal stabilizer for VR Camera, and $360^{\circ}$ Spherical VR bracket for six GoPro HERO4. The GoPro is small and weighs only $74 \mathrm{~g}$. This single-leg fixed accessory can easily fit on an unmanned aerial vehicle. The camera has a wide-angle lens that resembles a fisheye lens, obtaining an extended depth of field. The largest aperture is $\mathrm{f} / 2.8$, and the camera has a full $4 \mathrm{k}$ cinema resolution at $12 \mathrm{fps}$.
The aerial camera captured videos from different heights to determine the optimum height at which to shoot the video; the results of the experiment indicated that the optimum camera height was $500 \mathrm{~m}$, slightly higher than the clouds. Next, the panoramic view experiments were performed. An appropriate number of spatial samples were selected during photography to obtain highquality images. In the selected shooting area, the researchers avoided restricted flight areas and designed the image acquisition method according to the selected flight path. The flight path was controlled to record multiple photos at a selected angle. Typically, more than 40 images were captured, which were then combined in the post-processing step.

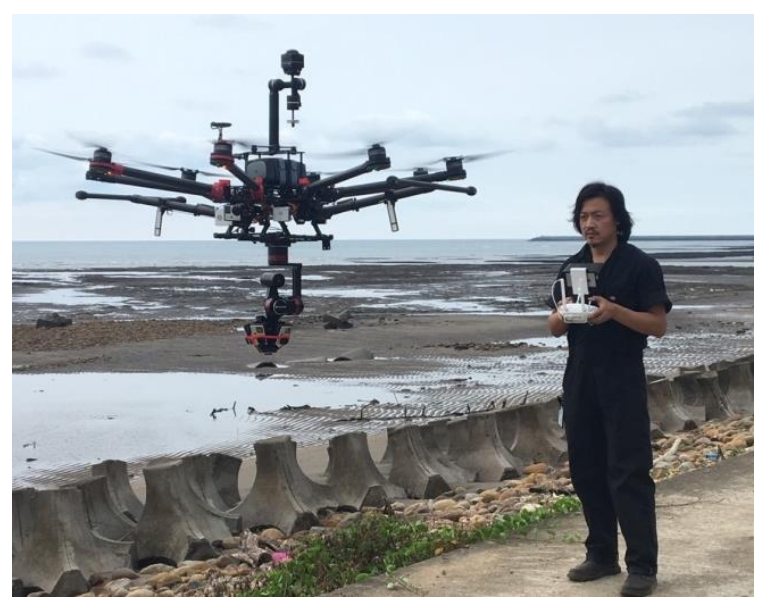

Figure 3: Hardware implementation and utilization.

\section{Petrochemical industrial areas}

\subsection{Spherical Transformation}

A spherical matrix operator was developed for panoramic image processing based on spherical panoramas. The photogrammetric equations were reviewed and matched with the geometry of spherical panoramic images. The shape of the line was a two-dimensional mapping of a three-dimensional space. The rotation matrix of spherical photogrammetry is an analytical approach that is used with spherical images [12].

The Laplacian operator is employed to obtain the relationship between omnidirectional panoramic and spherical images (tiny planet images).

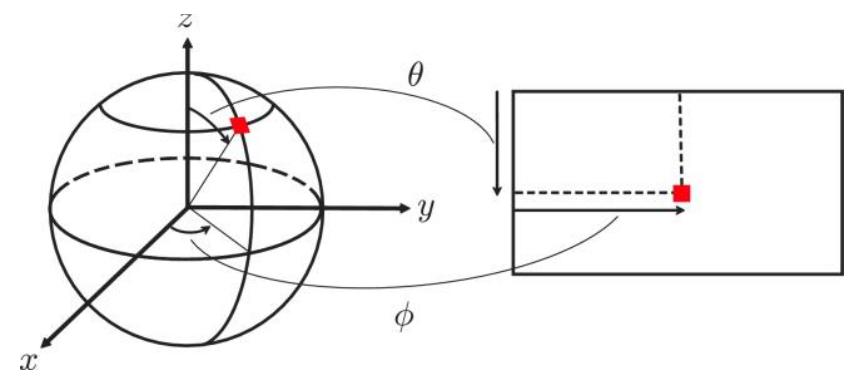

Figure 4: Coordinate the relationship between omnidirectional panoramic and spherical images [13].

An eight-neighbor Laplacian operator matrix has been reported for omnidirectional panoramic images. An extension of the Laplacian operator for omnidirectional panoramic images covering $360^{\circ}$ views solves the problem of nonuniformity of spatial resolution [13]. The spherical images were mapped by equirectangular projection in which the meridians and parallels are 


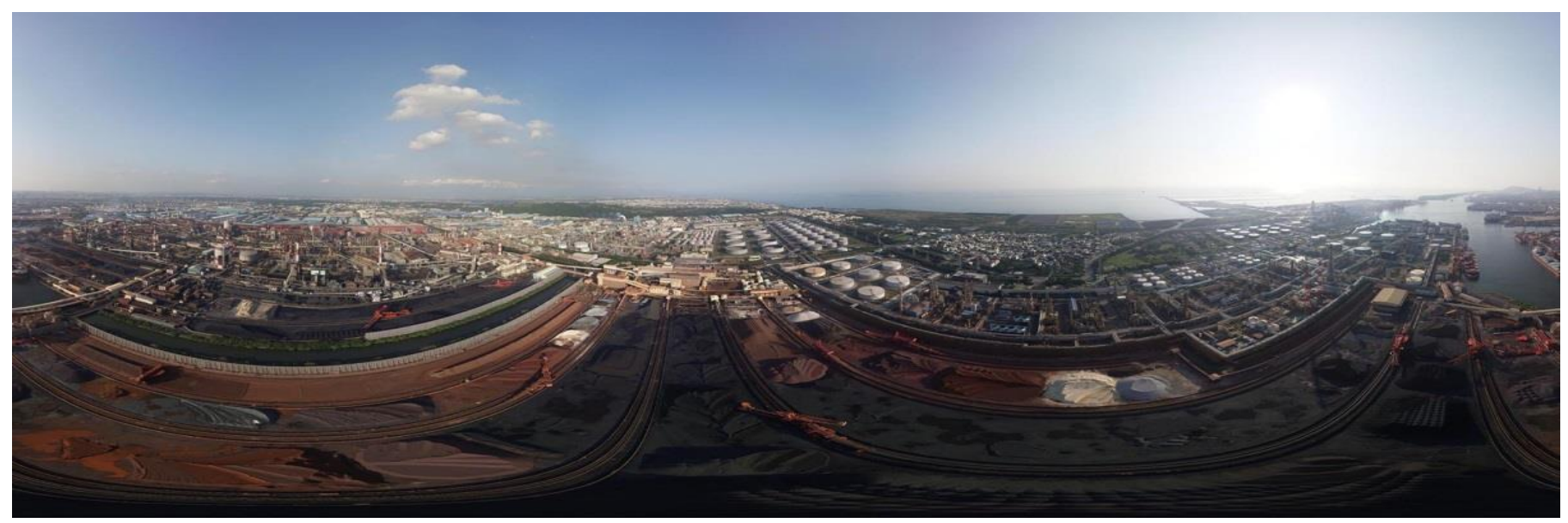

Figure 5: Panoramic aerial photo of Kaohsiung's Dalinpu village surrounded by the industrial zone.

represented by vertical and horizontal lines. The position of an image pixel related to a corresponding point on the spherical image. The computational time required (decomposition and integration processes) was considerably reduced by preparing a correspondence table of grid images in advance. This theoretical analysis considerably reduces the mathematical difficulties encountered by art producers.

We captured a top view of the industrial zone to trigger the viewer's thinking. The industrial area was shown in the images to appear calm in the morning (Figure 5). With spherical appearance, a tiny planet image is a metaphor and can prompt people to feel the pressure placed on the environment by the extensive industry comprising the tiny planet. The spherical projection viewer can then reflect on various appearances at different times (Figure 6).

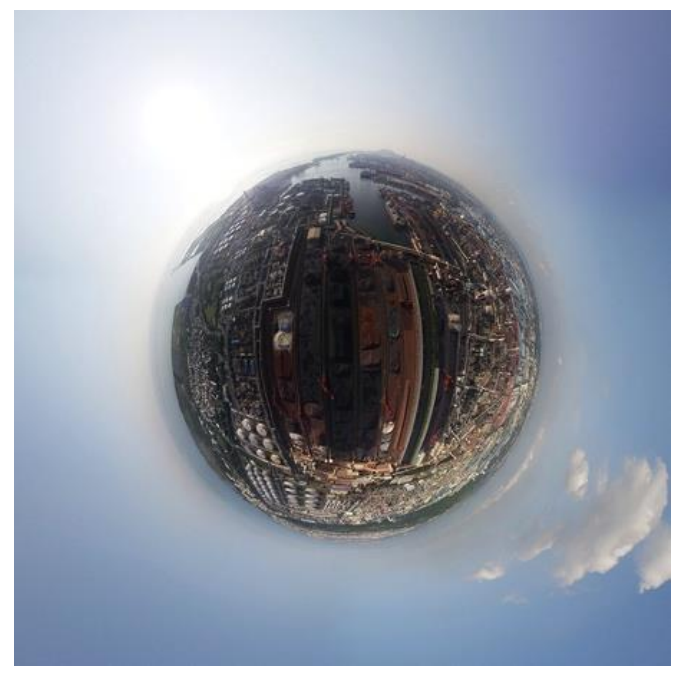

Figure 6: Spherical projection showing a tiny planet image.

\subsection{Dual-image Contrast}

The "double-sided" contrast image shows a sharp contrast between the industrialized world and untouched natural environment on the eastern and western coasts of Taiwan, respectively. The eastern coast is mountainous. From north of Hualien Creek to the south to Xiaoyeliu in Taitung County, facing the Pacific Ocean, the mountains, deep valleys, and coastal terrain are populated with indigenous people. Long-term differential erosion on the northern coast has created terraces and reef banks. Stones are scattered randomly over the coastline, showing pure and primitive beauty. Unusual geological patterns have been formed by geological movement, long-term weathering, and seawater erosion (Figure 7). The strata consist of interlayers of sandstone and shale from top to bottom.

The "two-sided" contrast image is a merging of the two hemispheres and shows the polarization between the industrial world and the untouched natural environment. Figure 8 presents a dual half-sphere image of the eastern region versus the western industrial zone. In a matrix developed for the region surrounding the factory, suspended particles and black smoke cover the original blue sky and white clouds. The blue sky and white clouds symbolize the need to breathe freely and deep inside desire. The matrix arrangement of industrial plants symbolizes regularity and suffocation, while the gray sky makes people feel oppressed.

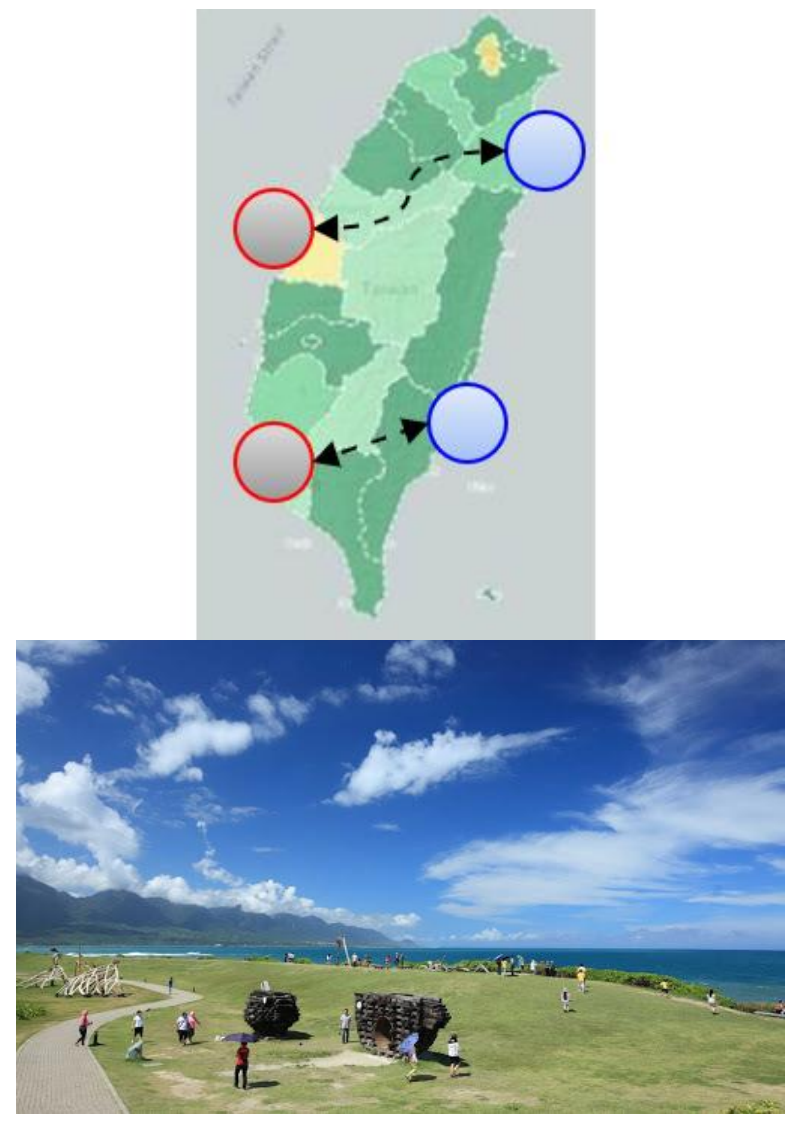

Figure 7: Contrasting locations on the eastern and western coasts of Taiwan 
Figure 9 shows a dual image of rocky topography in the northern region versus a dense industrial area on the western coast. When the two regions are merged in a single image, unevenness exists along the seam. In post-production, elastic zoom editing was employed to adjust the natural edges of the two images so that the seam is not apparent. The untouched sandstone coast intrigues the viewer and reminds them that it is an indispensable part of a sustainable life. The contrast between the natural etched space and the industrial area evokes strong reactions from the viewer.

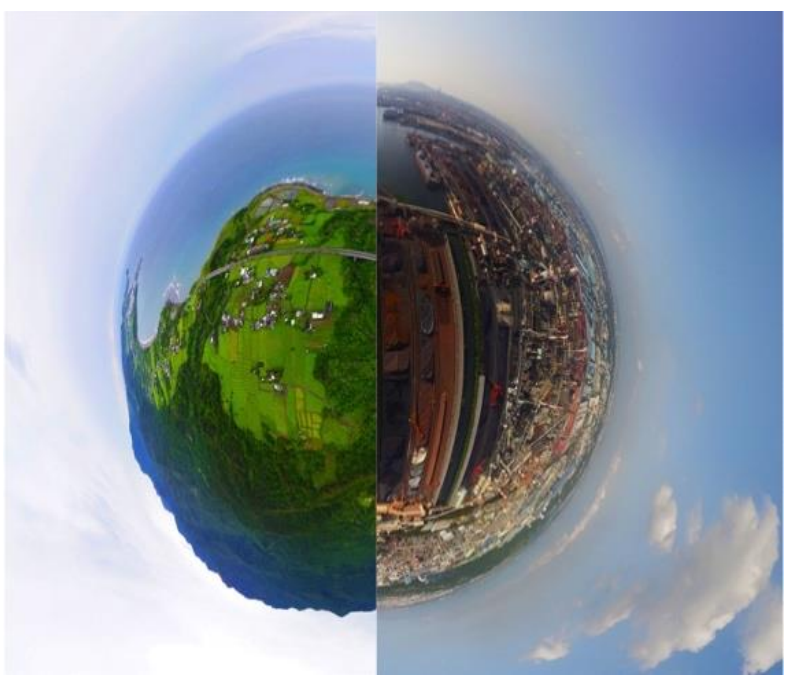

Figure 8: Green space in the eastern region versus an industrial zone in the west.

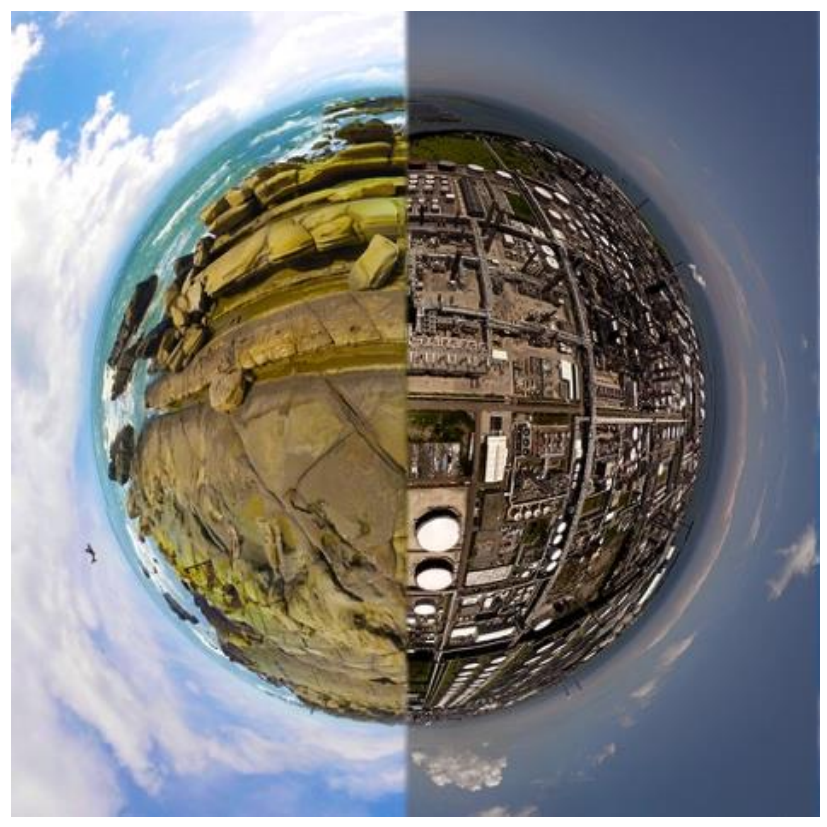

Figure 9: Dual images of rocky topography in the north versus a dense industrial area on the western coast.

\section{Thermal power plants}

\subsection{Smoky Tower}

The theme of the project was to convey the following: "God, please look at how the people have burned the planet you have created to satisfy their desires. Heaven or hell? Who can live in this place?" The film starts with the narration of a fictional ancient legend, reminding people that they should be in awe of nature, and shows a bird's-eye view of a soul floating in the sky, presenting a scene of overdevelopment of Taiwan's western coast industry. The monologue is written in a narrative style of mystery, with air pollution issues illustrated through aerial photography.

Incense burning is a traditional eastern custom but also associated with thermal power plants, towering columnar chimneys, and smoke. As the drone gradually approached the power plant, the first striking feature was numerous trenches containing burning coal. Symbolically, these trenches depict ashes formed by incense in exchange for satisfying human avarice. Figure 10 presents an image captured when the drone was at half the height of the chimney and depicted the chimney and other combustion units from a distance. The chimney is shown to be coated with images of animals (black-faced spoonbill birds) to confuse the public cognition. Finally, the drone reached the top of the chimney and surveyed the deep darkness, a strong symbolic representation of the endless desire for burning.

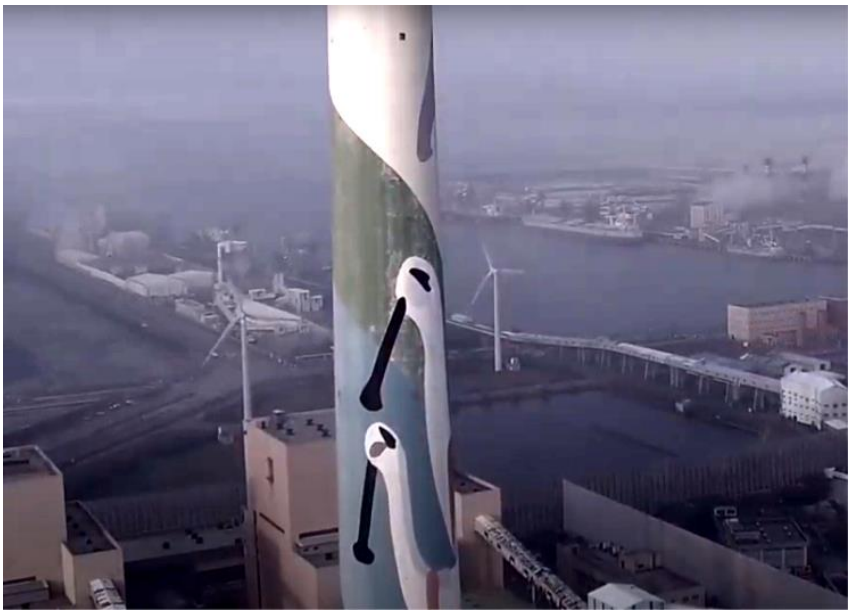

Figure 10: Chimney painted with images of animals [14].

\subsection{Panoramic Layout}

The natural coast of the east and industrial area of the west were combined to form a sharply contrasting image. The corresponding hemispheres on the left and right were inserted between the boundary; by overlapping the picture of the plane and 3D hemispheres. The combination of multiple post-production images increases the variability and also forms a sharp contrast between the blocks. This method of flipping and superimposing the images created the effect of alternation between heaven and earth (Figure 11).

In post-production, the bulge of the landscape was enhanced using a four-axis aerial camera passing through the clouds, creating a bird's-eye view (Figure 12). The images show the obstruction of the air in the sky, resulting in a heavy feeling. The cross-domain surreal composition encourages the viewer to engage in dialogue. The themes resonate with the audience and transform the interaction from a digital framework directly into the discussion on air pollution, from a perspective of environmental protection. Because no specific shooting space was used, the creator had to relinquish control of the visual frame, and the viewer is encouraged to discover for themselves. The design can be used to evoke empathy and ensure that the viewer has understood the message hidden in the image. 


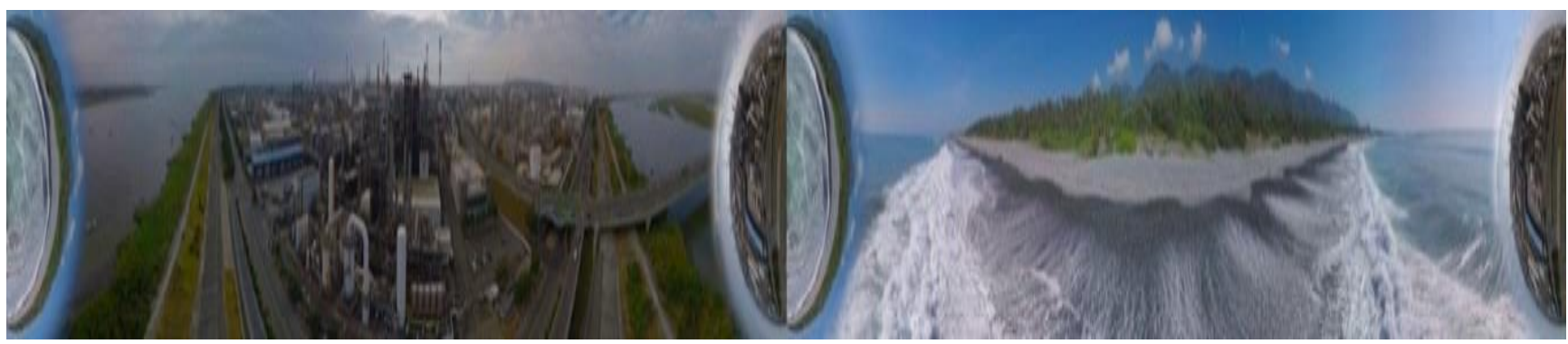

Figure 11: Comparison of an industrial area with a natural coastal beach [15].

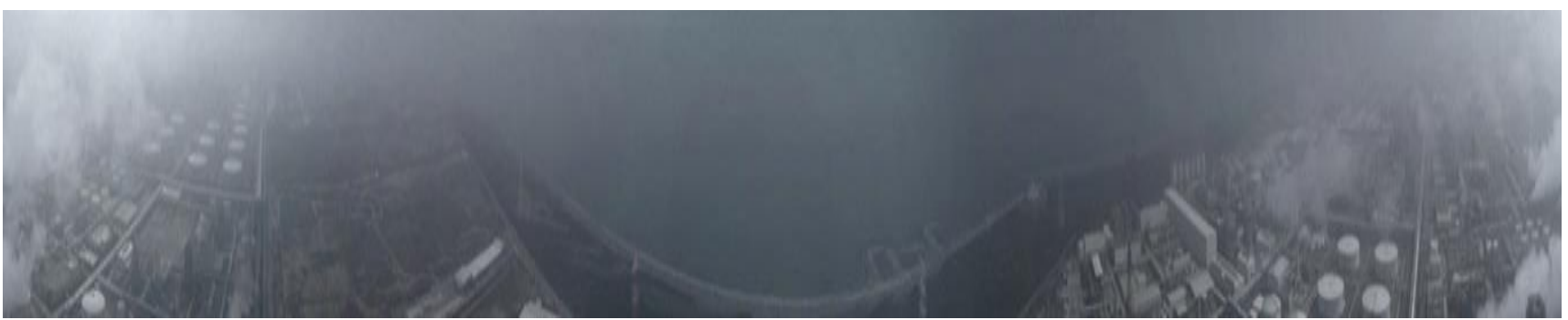

Figure 12: Bird's-eye view of severe air pollution, which blurs the view.

\section{Survey and discussion}

\subsection{Questionnaire and Analysis}

Thirty-seven college students were recruited for a survey. They were requested to watch the video on a VR headset connected to their smartphone. After watching the video, they completed a questionnaire, rating the video presentation, their level of feeling, and environmental concerns. We used SPSS software to analyze the participants' reported feelings. The responses to the unstructured questionnaire were then summarized. The content of the questionnaire is shown in below.

\section{Part A: Feeling (Likert scale)}

q1. The ground view is different from the ground.

q2. You feel yourself flying when watching the 360 film.

q3. I like to explore the environment in a 360-degree scene.

q4. Exploring the environment in 360 degrees is time-consuming.

q5. I will rotate my body to watch the rear during 360-degree exploration.

q6. I feel dizzy while watching.

q7. The way of viewing the left and right split screen is very creative.

q8. The spherical image that appears in the film helps to understand the theme of the film.

q9. The smog in the air is scary.

q10. I'm worried about air pollution in central Taiwan.

q11. I am willing to participate in PM2.5 environmental protection actions

q12. The visual communication technique is updated quickly, I can accept VR movies in the future.

Part B. Opinions on film creation:

o1. After watching the 360-degree image in the sky, my feeling is...

o2. The three chapters in the movie, which one do you like and why?

\subsection{Results}

The results of the 12-item narrative statistics are shown in table 1 . The results with the largest mean are questions 1 (4.5946), 12 (4.5405), and 5 (4.4054), respectively. This represents a strong agreement: the difference between watching in the air, movies that can already accept VR, will rotate the body to watch when watching with a mobile phone. Show that younger generations can accept VR skills and are willing to try them. The results with the smallest mean are questions 4 (2.8378), 6 (3.000), respectively. This represents disagreement: observation with a mobile phone is not time-consuming and no dizziness. This value shows the convenience of mobile phones to observe VR.

Table 1: Descriptive statistics of the items

\begin{tabular}{|l|l|l|}
\hline Items & Average & Std. deviation \\
\hline q1 & 4.5946 & 0.4977 \\
q2 & 3.5676 & 0.8673 \\
q3 & 4.0811 & 0.7218 \\
q4 & 2.8378 & 1.1184 \\
q5 & 4.4054 & 0.8963 \\
q6 & 3.0000 & 1.2693 \\
q7 & 3.8649 & 0.7875 \\
q8 & 3.4865 & 0.9013 \\
q9 & 3.6757 & 1.0015 \\
q10 & 4.2162 & 0.8862 \\
q11 & 3.9459 & 0.9703 \\
q12 & 4.5405 & 0.8365 \\
\hline
\end{tabular}

We merged three questions with similar meanings to understand the subjects' attitudes through the Bi-variable correlation analysis. Among them, t1 is the addition of three questions 1, 3, and 5, which means "like to experience 360-degree videos"; $\mathrm{t} 2$ is the question 9,10 , and 11 , and express willingness to do environmental protection. The participants all appreciated 
and wanted to watch the VR audiovisual presentation (Figure 13). The sounds in the film, such as the buzzing of the factory and sounds of the wind and heartbeat, helped them explore the content of the film. The audience was deeply affected by the film and reported that they were more likely to participate in environmental protection activities (Figure 14). We used an independent sample T-test to test whether male and female participants felt differently about the above issues; there were no significant difference.

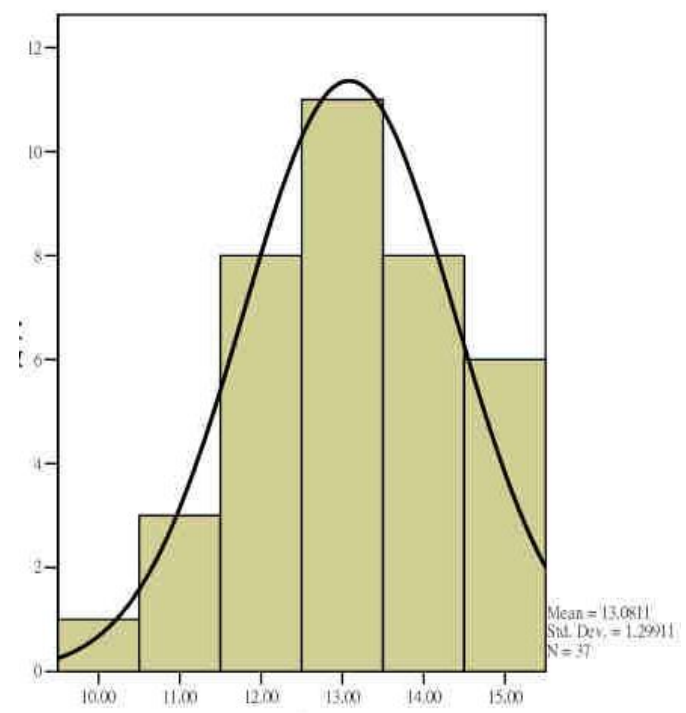

Figure 13: Histogram of participants' reactions (t1) to the film.

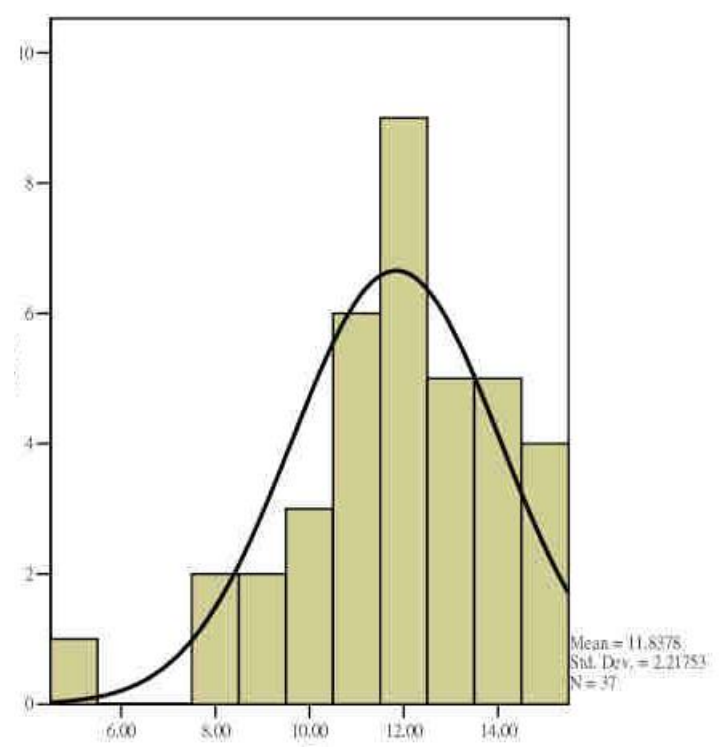

Figure 14: Participants' willingness to participate (t2) in the conservation event.

The participants reported that the images of a peaceful offshore fishing port surrounded by dense factories were depressing; the balance between the environment and factories was crucial. The smoke from the factory-made them feel as if they had entered the chimney and caused anxiety about the industrial areas scattered along the coast of Taiwan. The participants also reported that the view from the sky was very different from the view in their daily experience. The left versus right image segmentation produced a sharp contrast; the spherical display was creative, easy to understand, and added tension.
We use text analysis to count and observe their individual independent opinions. Among them, the count number of "willing to try to use VR" is 5, but "I don't know where to watch in the movie" and "feel tired or exhausted," still have eight counts. It shows that VR does not have a special visual focus so that people often enter a fantasy state without knowing where to look during exploration. This requires strengthening guidance in the design. The novelty feeling of flying in high altitude (skip from the existing state) and enter a broader experience counted 14 times, which shows the strong willingness to use this media.

\section{Conclusions}

Young students had a strong willingness to use VR media. Mobile phones perform well in changing the viewing angle for VR experiences. The "double-sided" contrast image shows a sharp contrast between the industrialized world and untouched natural environment on the eastern and western coastline of Taiwan. The $3 \mathrm{D}$ view of the digitally processed panoramic image enriches the spatial range of perception.

Post-production transformed the captured scenes and highlighted the contrast. The combined image also appeared on the east's natural coastline with the industrial landscape. Overlapping the planes in a 3D space evokes positive reactions from the viewer. Comparing natural space and an industrial area encourages the viewer to explore the differences between the two environments. The observation with a mobile phone is not time-consuming. The participants reported skipping from the present status and entering a broader experience.

\section{Conflict of Interest}

The authors declare no conflict of interest.

\section{References}

[1] T. Zhong, B. Mitchell, S. Scott, X. Huang, X., Y. Li, X. Lu,. "Growing centralization in China's farmland protection policy in response to policy failure and related upward-extending unwillingness to protect farmland since 1978.” Environment and Planning C: Politics and Space, 35(6), 1075-1097. https://doi.org/10.1177/0263774X16682958

[2] J. Y. Hsu, D. W. Gimm, J. Glassman, "A tale of two industrial zones: A geopolitical economy of differential development in Ulsan, South Korea, and Kaohsiung, Taiwan.” Environment and Planning A: Economy and Space, 50(2), 457-473, 2018. https://doi.org/10.1177/0308518X16680212

[3] R. Mabrook, and J. B. Singer, "Virtual Reality, $360^{\circ}$ video, and journalism studies: conceptual approaches to immersive technologies," Journalism Studies, 1-17, 2019. https://doi.org/10.1080/1461670X.2019.1568203

[4] E. Sirkkunen, and P. P. Rezaei, "Journalism in virtual reality: opportunities and future research challenges," In Proceedings of the 20th International Academic Mindtrek Conference, 297-303, ACM, 2016. https://doi.org/10.1145/2994310.2994353

[5] R. Chandra, Manohar Swaminathan, "Aerial imaging of a region using above ground aerial camera platform," US Patent Application 15/414,949, filed July 26, 2018. https://patents.google.com/patent/US20180213187A1/en

[6] S. Dorafshan, M. Maguire, V. Nathan, C. Coopmans, "Challenges in bridge inspection using small unmanned aerial systems: Results and lessons learned," In 2017 International Conference on Unmanned Aircraft Systems (ICUAS), 1722-1730, IEEE, 2017. https://doi.org/10.1109/ICUAS.2017.7991459

[7] DJI, https://www.dji.com, access date: 25 July, 2020, 12:20.

[8] Zuppinger-Dingley, Debra, Cornelia B. Krug, Owen Petchey, Bernhard Schmid, Norman Backhaus, and Michael E. Schaepman, "Editorial overview: environmental change issues: integrated global change and biodiversity research for a sustainable future," vii-xi, 2017. https://doi.org/10.1016/j.cosust.2018.05.019

[9] I. Budinska, "On Ethical and Legal Issues of Using Drones" In: Aspragathos N., Koustoumpardis P., Moulianitis V. (eds) Advances in Service and Industrial Robotics. RAAD 2018. Mechanisms and Machine Science, 67. Springer, Cham. 2018. https://doi.org/10.1007/978-3-030-00232-9_74 
W.Z. Feng et al. / Advances in Science, Technology and Engineering Systems Journal Vol. 5, No. 4, 849-856 (2020)

[10] Ippoliti, Elena, and M. Calvano, "Enhancing the cultural heritage between visual technologies and virtual restoration: case studies to models for visual communication," In Digital Curation: Breakthroughs in Research and Practice, 309-348, IGI. Global, 2019. https://doi.org/10.4018/978-1-52256921-3.ch015

[11] L. Brun, "Enabling 360 visual communications: next-level applications and connections," IEEE Consumer Electronics Magazine, 5(2), 38-43, 2016. doi: 10.1109/MCE.2016.2519050

[12] K. Hara, K. Inoue, and K. Urahama, "Gradient operators for feature extraction from omnidirectional panoramic images," Pattern Recognition Letters, 54, 89-96, 2015. https://doi.org/10.1016/j.patrec.2014.12.010

[13] G. Fangi and C. Nardinocchi, "Photogrammetric processing of spherical panoramas," The Photogrammetric Record, 28(143), 293-311, 2013. https://doi.org/10.1111/phor.12031

[14] https://www.youtube.com/watch?v=eFlfVw5A6XY, access date: 25 July, 2020, 12:25.

[15] https://youtu.be/z3IMRKr6iHw, access date: 25 July, 2020, 12:30. 